\title{
Lesiones físicas en accidentes de tránsito, en el cantón Cañar
}

\section{Physical injuries in traffic accident in the Cañar canton}

\author{
Yamasqui Padilla José Isidro \\ Carrera de Enfermería,Universidad Católica de Cuenca Extensión Cañar / Cañar,030201, Ecuador \\ *jiyamasquip@ucc.edu.ec
}

\begin{abstract}
Resumen
"Las lesiones físicas en accidentes de tránsito, en el cantón Cañar, período julio 2011 a junio 2012" han sido frecuentes ya sea por imprudencia e impericia de los conductores, por la irresponsabilidad de cada una de las personas al momento de sentarse frente al volante, faltas mecánicas de los vehículos y como también las inclemencias del tiempo; esto ha provocado lesiones físicas como: los politraumatismos, TEC, fracturas, entre otras; dejando a su vez secuelas que pueden ser temporales o permanentes, y con ello un alto costo no solo para la familia, sino también para con la sociedad y el estado. A través del presente estudio se busca que los conductores tomen conciencia de la gran importancia de asumir con absoluta responsabilidad esta tarea, para de esta manera salvaguardar lo más grandioso de la creación como es la vida de las personas; y lograr así disminuir las consecuencias fatales que puede causar la falta de responsabilidad al momento de sentarse frente al volante para conducir un automotor como son: accidentes de tránsito, lesiones físicas y sobre todo las secuelas que esto puede dejar. El objetivo general que persigue la presente investigación es: "Describir lesiones físicas en accidentes de tránsito, en el cantón Cañar, período julio 2011 a junio 2012”. La presente investigación se realizará en base al análisis de las historias clínicas de la oficina del SOAT, del Hospital Luis F. Martínez. Una vez obtenidos los resultados se harán las conclusiones respectivas y recomendaciones necesarias a las instituciones de tránsito y seguridad vial, y demás instituciones inmersas, para disminuir la incidencia de los accidentes de tránsito en este Cantón.
\end{abstract}

Palabras clave: Lesiones Físicas, Accidentes de Tránsito.

\begin{abstract}
The physical injuries in traffic accidents in the Cañar canton, period July 2011 to June 2012 which have been frequent either by negligence and malpractice of drivers, by the irresponsibility of each of the people when sitting behind the wheel mechanical vehicles and as inclement weather faults; this has caused physical injuries such as multiple trauma, TEC, fractures, among others; while leaving sequels that can be temporary or permanent, and thus a high cost not only for the family but also to society and the state. Through this study seeks drivers aware of the importance of assuming full responsibility this task, thus safeguarding the greatest of creation as is the life of people; and achieve thus reduce the fatal consequences that can cause lack of responsibility when you sit behind the wheel to drive a motor, such as: accidents, physical injuries and above all the consequences that this can leave. The overall objective of this research is: "Describe physical injuries in traffic accidents in the Cañar canton, period July 2011 to June 2012". This research was conducted based on an analysis of medical records of the office of SOAT, Hospital Luis F. Martinez. After obtaining the results the respective conclusions and recommendations will be given to the traffic institutions and road safety, and other institutions forming be made to reduce the incidence of traffic accidents in this Canton.
\end{abstract}

Key words: Physical Injury, Traffic Accident.

\section{Introducción}

Es importante el tema de investigación debido a que en el cantón Cañar, no hay un aporte o estudio científico que permita saber cuáles son lesiones físicas más frecuentes de los accidentes de tránsito y así disminuir los daños o lesiones que a menudo se ocasionan.

Son muchas las personas que mueren por año en accidentes de tránsito, pero son más los que sufren lesiones de distinta consideración. Ser víctima de un accidente implica sufrir lesiones que pueden demandar un largo período de recuperación o hasta dejar secuelas de por vida, puesto que los accidentes de tránsito constituyen un problema mundial por el impacto en la salud, los costos, y en especial porque está dentro de las 10 primeras causas de enfermedad y trauma.

En los últimos años con un parque automotor en crecimiento muy acelerado, donde los vehículos conducidos por personas que no tienen una preparación óptima para el manejo y respeto de las leyes de tránsito, con señales de tránsito escasas o poco visibles, la imprudencia de muchos 
conductores, el uso del celular durante el manejo, la ingesta de bebidas alcohólicas, entre otras, tiene un incremento preocupante de los accidentes de tránsito, lo que se refleja en las altas tasas de morbimortalidad de su población en los últimos años lo que amerita la realización del presente trabajo para conocer cuál es la lesión física más frecuente en los accidentes de tránsito en el cantón Cañar.

La organización mundial de salud estima que 1,3 millones de personas mueren por accidentes de tránsito cada año, de estos el $90 \%$ ocurre en países de bajos recursos y son los jóvenes de 15 a 29 años de edad, los más afectados. Entre 20 millones y 50 millones sufren traumatismos no mortales, y a su vez una proporción de estos padecen alguna forma de discapacidad. Las pérdidas económicas representan del 1 al $3 \%$ del producto interno bruto y en algunos casos puede llegar al $5 \%$. Si no se aplican medidas para evitarlo, se prevé que de aquí a 2020 los accidentes de tránsito causarán cada año 1,9 millones de muertes. Únicamente el $15 \%$ de los países cuenta con leyes completas relacionadas con cinco factores de riesgo: la velocidad excesiva, la conducción bajo los efectos del alcohol, el no uso de casco por los motociclistas, la utilización de los cinturones de seguridad y el empleo de medios de sujeción para los niños ${ }^{1}$.

A pesar de que los modernos vehículos automóviles van provistos de modo creciente de los medios de seguridad, están en constante revisión y cada vez más perfeccionados, elaborado por el genio inventivo y la técnica industrial. No obstante sigue aumentando sin cesar el número de accidentes y el derroche de vidas humanas por ellos producidas $^{2}$.

Por esta razón es fundamental utilizar correctamente los dispositivos de seguridad del automóvil: el cinturón de seguridad, el apoyacabezas y la posición correcta de la butaca para estar bien sentado frente al volante ${ }^{3}$.

En España, en el año 2008 se produjeron 93161 accidentes de circulación con víctimas (heridos o fallecidos), donde fallecieron 3100 personas, dentro de los 30 días siguientes al accidente y 130947 resultaron heridas ${ }^{4}$.

En los países de las Américas mueren cada año más de 130000 personas, más de 1200000 sufren heridas y cientos de miles quedan discapacitadas a consecuencias de las colisiones, choques o atropellamientos en las vías ${ }^{5}$.

En la Comunidad Andina los Accidentes de Tránsito, disminuyeron en un $13 \%$, en el año 2011, respecto al año anterior, al pasar de 319 mil en el año 2010, a 277 mil accidentes de tránsito en el año 2011. Heridos en accidentes de tránsito, en el año 2011, la comunidad andina registró 122 mil heridos en accidentes de tránsito, cifra que representó un incremento de 3 por ciento, respecto al año $2010^{6}$.

En el Ecuador según el INEC, estadísticas de transporte 2005, han existido 10697 víctimas de accidentes de tránsito, con 1387 muertes, 9098 heridos y 212 traumatismos. Y en la provincia del Cañar, aparecieron 276 víctimas de accidentes de tránsito, con 20 muertes, 256 heridos $^{7}$.

Todo lo señalado anteriormente dio las pautas necesarias para la elaboración del presente trabajo, ya que la presente investigación sirve de base para concienciar a las personas sobre lo grave y complicado puede ser víctima de un accidente de tránsito.

\section{Materiales y Métodos}

\subsection{Materiales}

Material. Historias Clínicas del "Hospital Luis F Martínez".

Lugar de la investigación. La presente investigación se realizó en el Cantón Cañar, Hospital Luis F. Martínez del Cantón Cañar.

El Cantón Cañar, tiene una Población, según el censo 2010 es de 59.323 habitantes y una extensión de 1.804 $\mathrm{Km} 2$, entre los sitios turísticos más importantes del país, destacándose entre otros la Fortaleza de Ingapirca y la Laguna de Culebrillas.

El Cantón Cañar, está situado al noroeste de la Provincia de Cañar, con una latitud de 3.160 m.s.n.m., con una temperatura 11.8 gradosC media anual, ubicado en la altiplanicie de la Cordillera de Los Andes, tiene una topografía accidentada ${ }^{8}$.

Según datos de la agencia provincial de tránsito del cantón Cañar tenemos un parque automotor de 4724 en el año 2010, en el 2011 existen 5708 y en el 2012 con 6663. Existiendo un crecimiento muy significativo del parque automotor.

Período de la investigación. El período de investigación fue desde julio del 2011 hasta junio del 2012.

Recursos empleados. Humanos y físicos:

1) Recursos Humanos

- El investigador

- Tutor

- Personal de estadística del hospital y la policía

2) Recursos Físicos

- Computador

- Impresora

- Hojas de papel bond

- Bolígrafos

- Cámara fotográfica

- Partes policiales

- Historias clínicas de la oficina del SOAT del Hospital Cantonal.

Universo Pacientes atendidos por accidentes de tránsito en el período comprendido desde julio del 2011 hasta junio del 2012 en el Hospital Luis F.

Muestra La muestra será los pacientes atendidos por accidentes de tránsito en el período comprendido desde julio del 2011 hasta junio del 2012 en la Oficina del SOAT del Hospital Luis F. Martínez Considerando los criterios de: 
1) Inclusión: Los pacientes atendidos por accidentes de tránsito en el período comprendido desde julio del 2011 hasta junio del 2012 en la Oficina del SOAT del Hospital Luis F. Martínez

2) Exclusión:

a) Todos los individuos que no sufrieron accidentes de tránsito.

b) Todos los individuos que no fueron atendidos en el Hospital.

c) Los que salen del período de estudio.

d) Todos los individuos que fallecieron.

\subsection{Métodos}

Tipo de investigación Estudio transversal, descriptivo de los pacientes que sufrieron accidente de tránsito, atendidos en el Hospital Cantonal "Luis F. Martínez", cuyos resultados se presentarán en tablas y la representación gráfica correspondiente.

Diseño de Investigación Análisis de las historias clínicas de la oficina del SOAT, del Hospital cantonal, artículos científicos, datos epidemiológicos, tesis, libros y revistas.

El trabajo estadístico se procesará en una base de datos, la misma que será presentada mediante tablas y gráficos con su respectivo análisis e interpretación.

\section{Resultados}

\begin{tabular}{|l|l|l|}
\hline Tip. de Lesion Fis. & Numero & Porcentaje( \%) \\
\hline TEC & 48 & $15.8 \%$ \\
\hline Politraumatismo & 37 & $\%$ \\
\hline Trauma abdominal & 10 & $3.3 \%$ \\
\hline Trauma de tórax & 16 & $5.3 \%$ \\
\hline Trauma cervical & 10 & $3.3 \%$ \\
\hline Trauma Facial & 12 & $4 \%$ \\
\hline Fractura & 33 & $10.9 \%$ \\
\hline Otros & 62 & $20.4 \%$ \\
\hline Total & 303 & $100 \%$ \\
\hline
\end{tabular}

Tabla 1. Lesión Física más frecuente en el período Julio 2011 - Junio 2012

Fuente: Datos obtenidos en el Hospital Luis F. Martínez Elaborado por: Dr. Isidro Yamasqui Padilla. La Figura 1 representa la red física de un ISP.

El tipo de lesión física más frecuente es el politraumatismo con un $37 \%$, seguido del TEC con un $15.8 \%$, con un 3,3\% trauma abdominal, un 5,3\% trauma de tórax, un $3,3 \%$ trauma cervical, un $4 \%$ trauma facial, un $10,9 \%$ fractura y un $20,4 \%$ otros.

\section{Discusiones}

En el presente período de estudio julio 2011 - junio 2012 , en primer lugar tenemos los politraumatismos con un $37 \%$, seguido de TEC con el $15.8 \%$, fractura $10.9 \%$, trauma facial $4 \%$, trauma de tórax $5.3 \%$, trauma cervical $3.3 \%$, trauma abdominal $3.3 \%$ y otros $20.4 \%$. Con un total de 303 víctimas de accidentes de tránsito, que representa el $100 \%$, del año de estudio.

En un estudio transversal en Argentina, las lesiones más frecuentes tenemos, traumatismo de cráneo 195 casos, traumatismo de miembros inferiores 226 casos, traumatismo de miembros superiores 67 casos, politraumatismos 63 casos Tesis doctoral, estudio médico legal de los cuadros lesivos en los accidentes de tráfico, la más frecuente es la cabeza, representando el 33,4\% del total, estas lesiones son muy variadas comprende desde contusiones a T.C.E. graves. Le sigue en frecuencia el tronco que representa el $26,2 \%$. El tercer lugar, lo ocupan las lesiones de miembros de miembros inferiores que asciende al 17,2

Estadísticas de accidentes de tránsito en el Ecuador durante el 2009 unas 14.869 personas resultaron heridas o discapacitadas en accidentes de circulación muchas de estas víctimas se podrían haber evitado con el uso del cinturón.

En el Ecuador según el INEC y estadísticas de transporte 2005, se han reportado 10697 víctimas de accidentes de tránsito, con 1387 muertes, 9098 heridos y 212 traumatismos. Y en la provincia del Cañar, se ha producido 276 víctimas de accidentes de tránsito, con 20 muertes, 256 heridos.

Haciendo un análisis de las literaturas antes descritas podríamos decir que la lesión más frecuente es el trauma craneoencefálico, los politraumatismos, trauma de miembros, fracturas, nuestro estudio difiere ya que en primer lugar están los politraumatismos seguidas de trauma craneoencefálico, y en tercer lugar las fracturas.

En el presente trabajo la hipótesis planteada fue que la lesión fisca más frecuente en el cantón Cañar es el trauma craneoencefálico, podemos decir que no se cumplió con la hipótesis, ya que en la presente investigación fue el politraumatismo en primer lugar seguido de trauma craneoencefálico. Ya que la literatura refieren que el TEC es la primera causa de accidentes de tránsito lo que no corrobora con nuestro estudio.
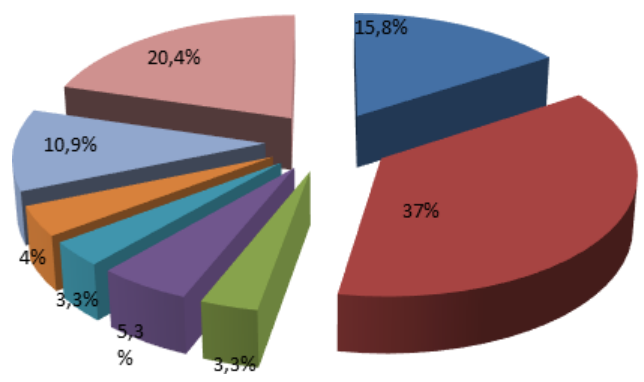

ITEC

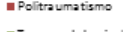

UT Truma abdomina

Trouma de tórax

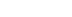

wotres

Fig. 1. Lesión física más frecuente Julio 2011 - Junio 2012 . Elaborado por Dr. Isidro Yamasqui Padilla. 


\section{Conclusiones}

Finalizado el presente estudio se puede concluir que del universo involucrado en la presente investigación la lesión fisca más frecuente es el Politraumatismo, seguida del TEC, la fractura, trauma de tórax, cervical y abdominal, entre las más importantes, esto debido a un parque automotor creciente, sin adecuada señalización vial, un bajo control de las autoridades , una baja concienciación de la ciudadanía, y al decir la impericia e imprudencia al momento de sentarse frente al volante, tengamos un alto grado de lesiones fiscas y lo más importante ser víctima de una secuela ya sea temporal o permanente, y en muchos de los casos lo difícil que se hace llevar esta secuela, además de los costos que representa para la familia la sociedad incluso para el mismo estado.

La presente investigación deja una puerta abierta para nuevos estudios, para así conocer si hemos mejorado es decir han disminuido los accidentes de tránsito y por ende las lesiones fiscas y sus secuelas.

\section{Agradecimientos}

Gracias a Dios por cada una de las oportunidades que me brinda, de manera muy especial al personal que labora en el Hospital Luis F. Martínez del cantón Cañar y a la Universidad Católica de Cuenca.

\section{Referencias}

1. R. De la Fuente, M. E. Medina-Mora, et al., "Las adicciones en méxico. i. el abuso del alcohol y los problemas relacionados," bi-digital, 2013.

2. G. Portero and P. Garamendi, "Reflexiones sobre la ley 34/2003 (2): Valoración de la tabla psiquiátrica," Cuadernos de Medicina Forense, no. 37, pp. 21-29, 2004.

3. V. Choquehuanca-Vilca, F. Cárdenas-García, J. Collazos-Carhuay, and W. Mendoza-Valladolid, "Perfil epidemiológico de los accidentes de tránsito en el perú, 2005-2009," Revista Peruana de Medicina Experimental y Salud Pública, vol. 27, no. 2, pp. 162$169,2010$.

4. E. J. Mejías, Estudio de la movilidad, accidentalidad y sus factores asociados en estudiantes de la Universidad de Granada. Editorial de la Universidad de Granada, 2011.

5. V. U. P. D. LA SEGURIDAD, “Organización panamericana de la salud," ORGANIZACIÓN PANAMERICANA DE LA SALUD, 2004.

6. G. Ferro and E. Lentini, "Infraestructura y equidad social: Experiencias en agua potable, saneamiento y transporte urbano de pasajeros en américa latina," cepal, 2012.

7. K. M. Encalada Reyes, Elaboración de un Plan de Negocios para la introducción del producto Tropiyogurt en la ciudad de Guayaquil. PhD thesis, ucsg, 2012.

8. V. INEC, "Censo de población y iv de vivienda, 1990," Provincia del Cañar, 1991.

Recibido: 01 de junio de 2016

Aceptado: 06 de mayo de 2017

Yamasqui José: Doctor en Medicina y Cirugia, Docente Universitario 